Artículos

\title{
El cerebro social y místico en el paciente dependiente de sustancias
}

\author{
The Social and Mystic Brain in the Substance Dependent Patient
}

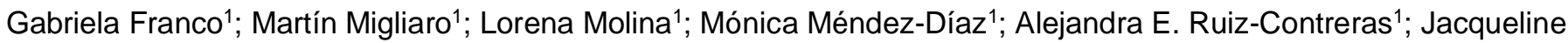
Cortés-Morelos ${ }^{1}$; Andrea Herrera-Solís ${ }^{1}$; Oscar E. Prospéro-García ${ }^{2}$
\end{abstract}

1. Universidad Nacional Autónoma de México

2. Hospital General Dr. Manuel Gea González

\section{Resumen}

El humano tiene un sistema cerebral que se activa cuando interacciona con otras personas que es el cerebro social, y otro cuando siente tener una experiencia mística que es el cerebro místico. Postulamos que ambos sistemas contribuyen a facilitar la integración del individuo a sus grupos de apoyo, y dicha integración le permite evitar o controlar trastornos psiquiátricos, como el trastorno de uso de sustancias (TUS). La falla en esta integración, como ocurre debido a una crianza negligente o abusiva, y/o viviendo dentro una red de apoyo social deficiente, vulnera al sujeto a padecer el TUS. En cuanto a la pertenencia a grupos religiosos, la observancia de su liturgia contribuye al bienestar físico y mental de los creyentes, resultando importante para prevenir el TUS y en muchos casos para rehabilitar al paciente que lo solicita. El objetivo de esta revisión es discutir las evidencias del involucro del cerebro social y del cerebro místico en la integración de una persona a sus grupos de apoyo, así como la falla de estos sistemas en conseguir dicho objetivo, como factores de riesgo para desarrollar o mantener trastornos psiquiátricos y el TUS.

Palabras clave: trastorno por uso de sustancias, crianza; red de apoyo social, religiosidad, adicción

Gabriela Franco. Hospital Psiquiátrico Fray Bernardino Álvarez Grupo de Neurociencias de la Universidad Nacional Autónoma de México.

Martin Migliaro. Laboratorio de Cannabinoides, Departamento de Fisiología, Facultad de Medicina. UNAM. https://orcid.org/0000-0002-3988-3838

Lorena Molina. Laboratorio de Cannabinoides, Departamento de Fisiología, Facultad de Medicina. UNAM. Mónica Méndez-Díaz. Laboratorio de Cannabinoides, Departamento de Fisiología, Facultad de Medicina. UNAM. http://orcid.org/0000-0002-0949-3070,

Alejandra E. Ruiz-Contreras. Laboratorio de Neurogenómica Cognitiva, Coordinación de Psicobiología y Neurociencias. UNAM.

http://orcid.org/0000-0002-9829-7636

Jacqueline Cortés - Morelos. Departamento de Psiquiatría y Salud Mental, Facultad de Medicina. UNAM. Andrea Herrera-Solís. Laboratorio Efectos Terapéuticos de los Cannabinoides, Subdirección de Investigación Biomédica, Hospital General Dr. Manuel Gea González.

https://orcid.org/0000-0001-7400-3325,

Oscar E. Prospéro-García. Laboratorio de Cannabinoides, Departamento de Fisiología, Facultad de Medicina. UNAM.

http://orcid.org/0000-0003-4936-3139

Autor para correspondencia: Oscar E. Prospéro-García opg@unam.mx 


\begin{abstract}
Human beings activate a neural system when involved in social interaction known as the social brain, and similarly, mystic experiences stimulate the mystic brain. We postulate that both systems help humans to integrate into their support group, that integration allows them to avoid or control psychiatric disorders, and to reduce the risk of developing a substance use disorder (SUD). When this integration fails, as consequence of child abuse, negligence, and/or a poor social support network, subjects are more vulnerable to suffer a SUD. On the other hand, the belongingness to religious groups helps to maintain physical and mental well-being of believers, hence, preventing the development of SUD or rehabilitating the subject after suffering from it. Therefore, the aim of this review is to discuss the role of the social and mystic brain in individual integration to a social support group and how the failure of these systems represents a risk factor for developing or maintaining psychiatric disorders and SUD.
\end{abstract}

Key words: substance use disorder, child rearing, social network, religion, addiction interactions

DOI https://doi.org/10.36793/psicumex.v11i1.393

Recibido 17 de Septiembre de 2020

Aceptado 14 de Agosto de 2021

Publicado 26 de Noviembre de 2021 


\section{Introducción}

Los humanos, al igual que otras especies animales, desde el nacimiento necesitan de la convivencia con sus conespecíficos, de lo contrario no sobrevivirían (Cacciopo y Patrick, 2008). La teoría del cerebro social (Dunbar, 2009) ha propuesto que, evolutivamente, la habilidad de los humanos de establecer lazos afectivos se ha conservado dado que, en el pasado, quienes lograban sobrevivir eran aquellos que vivían en grupos. Incluso se ha dicho que el lenguaje permitió al Homo sapiens una ventaja definitiva sobre el Homo neanderthalensis en la sobrevivencia. Gracias a que nuestros ancestros crearon lazos afectivos entre sí, lograron formar grupos para cazar a grandes animales, recolectar comida, domesticar el fuego, cocinar para reducir el tiempo de digestión y obtener el máximo beneficio de los alimentos en el menor tiempo posible, perfeccionar las técnicas para la construcción de herramientas, crear la agricultura, la domesticación de los animales, y el arte y la cultura, entre otras cosas, y con ello enfrentar las presiones del medio ambiente con éxito y sobrevivir.

Sin embargo, no es suficiente sobrevivir al predador, también es necesario tener salud y bienestar. Para ello, se requiere de varios reforzadores, entre los que se incluye tener interacciones psicosociales y lazos afectivos duraderos con otras personas. Es decir, una red de apoyo, de tal forma que el sujeto sienta seguridad y satisfacción, lo que le ayuda a contender exitosamente con las demandas del medio. Para el humano, así como para todos los animales altriciales, una red de apoyo debe brindar protección desde antes que el individuo sea capaz de solicitarlo.

En los humanos, los primeros años de vida son etapas clave en el desarrollo del sistema nervioso central (Stiles, 2017). El cerebro sigue madurando hasta los 30 años y es la interacción con sus conespecíficos en gran medida lo que lo hace madurar (Petanjek et al., 2011; Vygotsky, 1995). Las experiencias que una persona va teniendo en la vida provocan cambios en la expresión de los genes (epigénesis) que a su vez genera cambios en la sinaptización y, por lo mismo, en la función de los sistemas cerebrales (McEwen, 
2016). Se puede decir que la historia de vida de una persona está escrita en su conectoma (conjunto de sinapsis), lo cual se puede finalmente observar en su conducta. Las vivencias que un sujeto tiene a lo largo de su vida, el ambiente en el que crece y sus interacciones psicosociales lo ayudan a generar un altruismo recíproco, así como lazos de lealtad y amistad que forma con su familia y sus amigos (moldeamiento social). Todas estas interacciones tienen un impacto en el cerebro del sujeto y en su manera de percibirse a sí mismo, de contender con su medio ambiente y en sus expectativas del futuro (Bifulco et al., 1991; Blakemore, 2010).

En esta revisión narrativa abordamos los sistemas cerebrales más estudiados en el origen de la adicción a sustancias, las interacciones psicosociales positivas y negativas, la religiosidad como una forma de expresión del misticismo y el involucro de todos estos sistemas en la generación de una persona adicta, así como en su rehabilitación.

\section{El desarrollo del individuo}

El Fondo Internacional de Emergencia de las Naciones Unidas para la Infancia (UNICEF, 2019) establece 10 derechos básicos que los grupos deben ofrecer a los niños:

1. Derecho a la igualdad sin distinción de raza, religión o nacionalidad. 2. Derecho a una protección especial para que puedan crecer física, mental y socialmente sanos y libres. 3. Derecho a tener un nombre y una nacionalidad. 4. Derecho a una alimentación, vivienda y atención médica adecuadas. 5. Derecho a la educación y a atenciones especiales para los niños y niñas con discapacidad. 6 . Derecho a comprensión y amor por parte de las familias y las sociedades. 7. Derecho a una educación gratuita. Derecho a jugar y divertirse. 8. Derecho a recibir atención y ayuda preferentemente en casos de peligro. 9. Derecho a ser protegido contra el abandono y el trabajo infantil. 10. Derecho a recibir una educación que fomente la solidaridad, la amistad y la justicia entre todo el mundo.

El cumplimiento voluntario por parte de los padres o cuidadores primarios de estos 10 derechos básicos, o de la mayoría de ellos, se considera un cuidado óptimo, mientras que su incumplimiento voluntario se considera cuidado negligente. En la adolescencia hay cambios estructurales en el cerebro, que son muy 
dinámicos y, por lo mismo, terreno fértil para generar un adulto imaginativo y creativo (Blakemore et al., 2007) o para afectarlo de una manera que interfiera con el desarrollo de estrategias que lo adapten a su grupo, por lo que su integración al mismo se ve comprometida. Para generar una estrategia adaptativa, el cerebro se modifica de manera que sus neuronas aumentan la eficiencia con que se comunican entre sí, optimizando ensambles neuronales funcionales (Asok et al., 2019; Hebb, 1949). Es decir, las neuronas que disparan juntas fortalecen su interacción. Esto es a lo que llamamos una sinapsis de Hebb o hebbiana (Hebb, 1949). Estas redes neuronales, ya refinadas durante la adolescencia, son las que subyacen a las habilidades físicas y mentales del adulto. El niño se transforma en adolescente, entre otras muchas cosas, porque tiene un cerebro que ha generado nuevas sinapsis entre sus neuronas (Petanjek et al., 2011). Este abundante conectoma representa posibilidades de aprendizaje en el adolescente que prevalecerán en el adulto. Muchas de estas sinapsis desaparecerán hacia la mitad de la adolescencia (Gogtay et al., 2004), pero las que se quedan son las que están fortalecidas. Las hebbianas le serán útiles para las actividades que le permitirán llevar una vida adulta creativa y productiva si el ambiente fue amigable; pero si el ambiente fue hostil, es posible que su creatividad la dedique a generar estrategias de defensa y ataque, ya que así ha aprendido a contender con las presiones del medio. Por ello, la niñez y la adolescencia son momentos en que la red de apoyo del adolescente debe mostrarle posibilidades de desarrollar sus habilidades físicas y mentales en actividades que lo ayuden a crecer mental y emocionalmente, y que terminen haciendo de él un adulto integrado a su grupo y útil a su sociedad. Los grupos disfuncionales entorpecen este tipo de crecimiento, fortaleciendo la percepción hostil del ambiente y la incertidumbre del futuro (Anda et al., 2006), propiciando cambios epigenéticos que fortalecen conductas antisociales. No obstante, es importante enfatizar que, para que se establezcan y desarrollen interacciones sociales, la evolución ha favorecido la especialización de un sistema cerebral al cual llamamos cerebro social que regula la cognición social. 


\section{Cognición social}

La cognición social se refiere a la capacidad para dar sentido a las intenciones y conductas de los conspecíficos a través de las señales que generan (Bandura y Walters, 1974; Frith y Frith, 2007). Esta habilidad requiere del concurso de varios procesos cognitivos que permiten entender a los demás para interaccionar con ellos. Esto incluye el procesamiento de caras, movimientos, posturas, actitudes y gestos que ayudan a predecir las conductas que el interlocutor exhibirá en respuesta a la conducta del ego (Den Ouden et al., 2005; Farroni et al., 2005).

Así, gracias a la cognición social, podemos inferir las intenciones de una persona o de un grupo a través de su conducta. Leemos el lenguaje no verbal, inferimos si las intenciones del otro son amigables u hostiles a partir de los movimientos de su cuerpo (Den Ouden et al., 2005). Asimismo, cuando estamos en una interacción uno a uno, podemos ver los ojos del interlocutor, v.gr., ojos entrecerrados, la curvatura de las cejas, ceño fruncido, la posición de sus labios, labios apretados, la dilatación de las fosas nasales y, observando esto, podemos inferir lo qué está pensando. A esta habilidad la llamamos teoría de la mente (TOM) o mentalización (Lewis et al., 2017).

\section{Neuroanatomía del cerebro social}

Este sistema cerebral, del que depende la cognición social y la TOM, está conformado por la corteza prefrontal dorsal medial (CPFdm), el surco temporal superior posterior (STSp), la unión temporo-parietal (UTP), la corteza temporal anterior (CTA) y la circunvolución frontal inferior (CFI); y se activa en condiciones en las que el sujeto está llevando a cabo interacciones psicosociales (Blakemore y Frith, 2004; Lewis et al., 2017). Asimismo, se ha involucrado al lóbulo de la ínsula (LOBIN) en los procesos de empatía emocional; y a las neuronas espejo, en mecanismos de aprendizaje vicario (imitación) (Rizzolatti y Sinigaglia, 2016). Todas estas observaciones describen la activación de las áreas del cerebro mencionadas 
cuando interactuamos con conspecíficos y, por lo mismo, sustentan la existencia de un cerebro social que coordina la interacción de los grupos y participa en la creación y fortalecimiento de vínculos sociales.

Las emociones asociadas a las interacciones psicosociales que coordina el cerebro social son amplificadas por dos sistemas cerebrales que conocemos como el de motivación y recompensa, y el de defensa (ver Fig. 1).

\section{Figura 1}

Áreas cerebrales del cerebro social, cerebro místico, sistema de motivación y recompensa, y del sistema de defensa

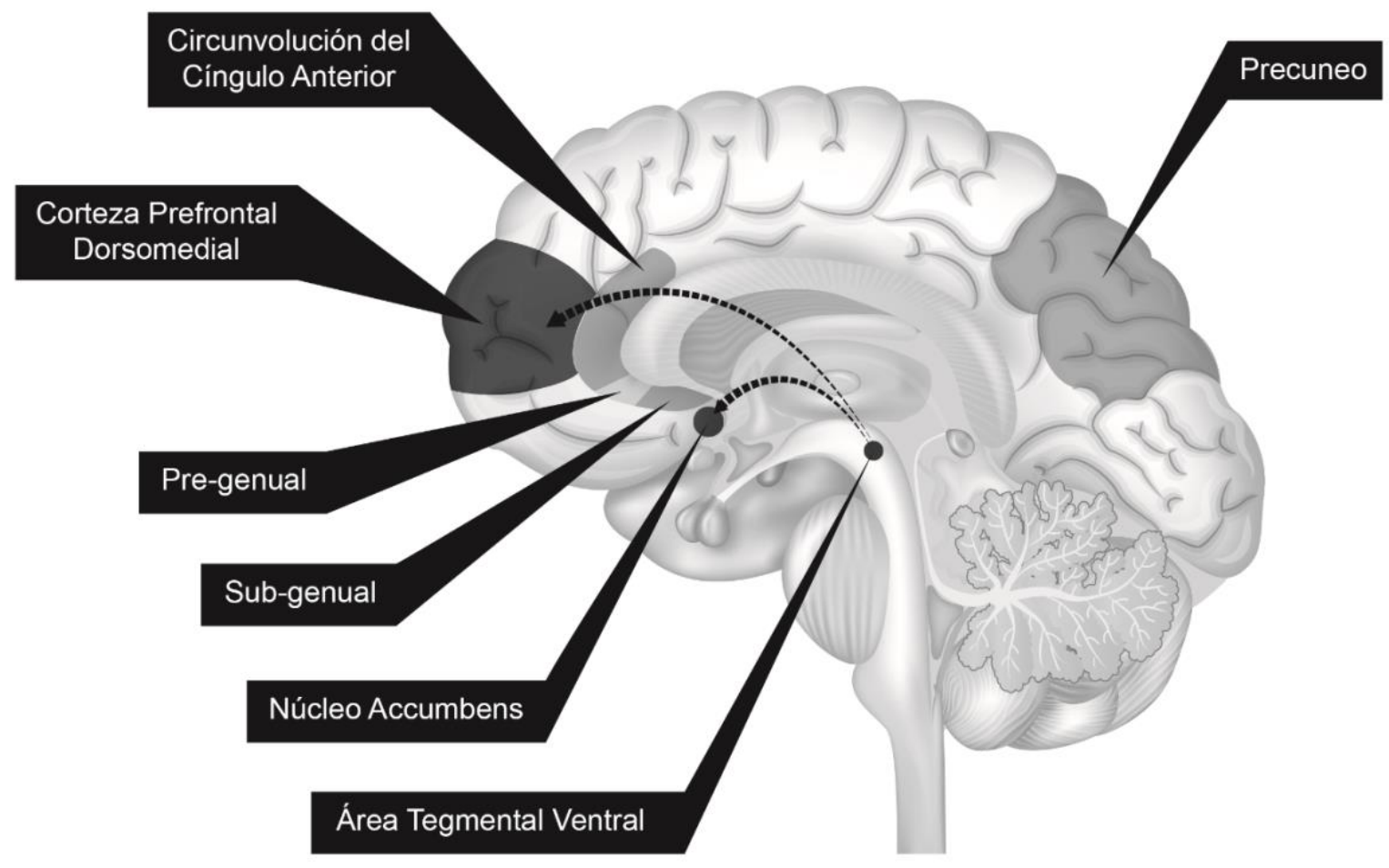




\section{El sistema de motivación y recompensa}

Este sistema es el que se activa cuando llevamos a cabo una conducta que nos produce placer, por ejemplo, comer o beber agua. Asimismo, la anticipación del placer y la interacción psicosocial lo activa. La interacción psicosocial puede buscar la integración del individuo al grupo, por lo que puede facilitar la deseabilidad social, que no necesariamente es placentera, pero sí su resultado, que puede ser la aceptación por el grupo (King y Bruner, 2000; Stodel, 2015). Estar con los amigos o la familia, con la pareja o estar observando jugar a nuestro equipo de futbol favorito también activa a este sistema. Los eventos no gratificantes, como comer algo desagradable o interaccionar con una persona con la que no simpatizamos, no activan a este sistema, sino al de defensa (Vicario et al., 2017) que explicaremos adelante. El sistema está dividido al menos en dos partes funcionales: la motivación y la recompensa. La primera cumple con la función de hacernos sentir deseo por un reforzador y está generada por el área tegmental ventral lateral (ATV1), y la amígdala (AMY), las cuales proyectan al núcleo accumbens (NAc). El ATV estimula al NAc con dopamina y la AMY con glutamato. También el núcleo cama de la estría terminal (NCST) interviene proyectando a la ATV, facilitando su actividad, inhibiendo a las neuronas GABAérgicas locales del ATV (Ver Prospéro-García et al., 2021). Esta combinación de actividades de dichas estructuras hace que la motivación sea una sensación subjetiva de displacer. El hambre, la sed, el deseo sexual y el de dormir son básicamente emociones de displacer, que sólo la obtención del reforzador mitiga. Asimismo, la ausencia prolongada de familiares o amigos nos motiva a buscarlos. Complementariamente, la obtención del reforzador produce una recompensa. El reforzador puede ser una conducta como la sexual, el consumo de alimento, la interacción social positiva como ver a la familia, los amigos, conocer nuevas personas o iniciar una relación amorosa; hace que el sistema etiquete la conducta como gratificante, por lo que desearemos volver a realizarla. En este proceso de gratificación, la ATV medial, la corteza prefrontal medial (CPFm) y el precúneo parietal son cruciales. 
De acuerdo con Taleb y Douadi (2012), existen en economía tres sistemas funcionales: el sistema frágil, el robusto y el antifrágil. El sistema frágil es aquel que sucumbe rápidamente ante la acción de un estímulo determinado, resultando dañado o destruido. El sistema robusto es el que la acción de un estímulo determinado lo dañará o destruirá, si es que lo logra, después de ejercer su acción sobre él por mucho tiempo. Finalmente, el sistema antifrágil es aquel que se fortalece ante la presencia de un estímulo que es dañino para los otros dos sistemas en condiciones semejantes. Dicho estímulo lo vuelve más eficiente (Taleb y Douady, 2012). En este contexto, el sistema de motivación y recompensa es frágil, ya que la repetición del mismo estímulo reducirá su respuesta de una manera no lineal. Por ejemplo, comer una porción de alimento reduce la probabilidad de disfrutar una segunda porción, o una tercera.

Es importante detectar también estímulos potencialmente adversos. Una gran variedad de respuestas que el humano exhibe son las que llamamos reforzamiento negativo. Consiste en conductas evitativas y motivación para buscar refugio y apoyo (Cannon, 1914). Prácticamente, todo ser viviente usa este tipo de reforzamiento, como la Aplysia califórnica entrenada por Kandel y Schwartz (1982) para que evitara choques eléctricos. Así que mientras el sistema de recompensa promueve la aproximación e interacción con el reforzador, el sistema de defensa evita al reforzador, que en este caso llamamos estresor (Grupe y Nitschke, 2013).

Existe una interacción recíproca entre los sistemas de motivación, recompensa y defensa, de tal manera que cuando se activa uno el otro reduce su actividad (Solomon y Corbit, 1974).

\section{El sistema de defensa}

Llamamos a este sistema de defensa, ya que es el que se activa cuando detectamos un estresor cuya proximidad es inmediata y nos produce miedo (Grupe y Nitschke, 2013). Este sistema nos ayuda a crear estrategias que nos permiten la huida o defensa (Cannon, 1914), la búsqueda de refugio y/o apoyo (Berridge, 2018). También se activa cuando el estresor aún no está próximo, no se conocen sus características 
particulares ni se sabe con precisión cuándo se presentará, pero se anticipa que será pronto. Así que, la incertidumbre sobre las características específicas del estresor produce ansiedad y ayuda a generar un plan de acción diseñado con base en la probabilidad de ocurrencia y de las potenciales características que exhiba el estresor (Grupe y Nitschke, 2013). Si el estresor tiene características que rebasan nuestros recursos, es una amenaza, en ese caso, el plan está orientado a la reducción del daño; pero si el estresor no rebaza nuestros recursos, entonces el plan se genera con la intención de neutralizarlo. Dentro de las estructuras que forman este sistema están la AMY, el NCST, la circunvolución del cíngulo anterior (CCA), el LOBIN, el núcleo paraventricular hipotalámico (PVN) y el sistema nervioso autónomo (Craske et al., 2017). Este sistema de defensa, al contrario del sistema de motivación y recompensa, es antifrágil (Taleb y Douady, 2012). Es decir, el sistema responde de manera no lineal, más robusta y eficiente conforme se presenten los estímulos. La presencia de los estímulos negativos lo fortalecen. Una persona que haya sufrido un evento traumatizante responderá con mayor intensidad a estresores semejantes o similares, ya que generaliza la respuesta (Prospero-García et al., 2021).

\section{La interacción del cerebro social con el sistema de motivación y recompensa, y con el sistema de defensa}

La cognición social y la TOM, reguladas por el cerebro social, permiten la detección de emociones con posturas, movimientos y expresiones faciales. Desde los trabajos de Paul Ekman (Ekman, 1993) sabemos que las expresiones faciales asociadas a emociones son universales, por lo que el cerebro social las reconoce y actúa en consecuencia. Las expresiones faciales amigables, v.gr., las comisuras de los labios y las mejillas se elevan (una sonrisa), los ojos se achican, y el pulgar del interlocutor posicionado para arriba en señal de aprobación activan al sistema de motivación y recompensa (Kohls et al., 2013), causando en la persona la sensación subjetiva de placer. Mientras que si lo que ve es una persona con el ceño fruncido, las comisuras de los labios estiradas de tal manera que se vean los dientes, las fosas nasales dilatadas, el cuerpo 
firme y los puños cerrados, entonces el sistema de defensa genera una sensación de miedo o ansiedad (Critchley y Garfinkel, 2014). Simplemente detectar en un grupo quién es el líder o jefe hace que la amígdala se active (Kumaran et al., 2012).

Es posible que la persona no sea muy hábil en reconocer una emoción y confunda las expresiones faciales, por ejemplo, que la expresión de una persona preocupada la confunda con enojada. Si es el jefe, la persona piensa que está enojado con él y que es posible que lo regañe o lo despida. Esta es una distorsión cognitiva (Beck, Rush, Shaw y Emery, 1979); es decir, una interpretación errónea de la expresión del jefe, tomando por enojo lo que realmente es preocupación. De cualquier manera, la emoción negativa evocada por esta distorsión cognitiva está modulada por el sistema de defensa.

\section{El grupo y la aceptación del individuo}

Es evidente, entonces, que las experiencias sociales positivas o negativas tienen un impacto sobre el cerebro social, la conducta y la persona como sujeto social. Por consiguiente, podemos sugerir que las experiencias positivas vuelven al individuo leal y solidario con el grupo; mientras que las negativas lo vuelven desconfiado, agresivo, intolerante y vulnerable al uso de sustancias. Por ejemplo, la Oficina de las Naciones Unidas contra la Droga y el Delito (UNODC, 2018) indicó que las experiencias sociales adversas que están más relacionadas al consumo de sustancias son la crianza negligente y/o los abusos físicos, verbales o sexuales durante la infancia, los cuales tienen un impacto negativo sobre las estrategias de integración del sujeto a su grupo, por lo que será rechazado y su salud mental se verá afectada, generando con frecuencia ansiedad y depresión (Heim et al., 2010). Estas respuestas creemos que están mediadas por el sistema de defensa, el cual se fortalece con estas experiencias debido a su naturaleza antifrágil (Prospero-Garcia et al., 2021). De esta manera, en el futuro responderá con mayor eficacia a los estímulos negativos y hará que el sujeto con frecuencia, si no siempre, esté ansioso y posiblemente deprimido (Heim et al., 2010). En esta condición, la probabilidad de desarrollar un consumo descontrolado de drogas que puede progresar a su 
dependencia aumenta (Conway et al., 2006; Regier et al., 1990). Las drogas, en este caso, ayudan a mitigar la hipersensibilidad del sistema de defensa, por lo que reducen la ansiedad y mejoran la depresión de la persona. Es importante resaltar, sin embargo, que hay personas que desarrollan estrategias adaptativas que les permiten contender con la adversidad y tener un grupo de apoyo que los fortalece y los ayuda a ser personas de éxito social; es decir, exhiben longanimidad (resiliencia) ante la crianza negligente o abusiva, reduciendo la probabilidad de consumir de manera descontrolada sustancias.

El análisis del impacto del estrés psicosocial, por ejemplo, la demanda del cumplimiento de tareas que creemos que sobrepasan nuestros recursos cognitivos, físicos y/o económicos, en la generación de síntomas psiquiátricos se ha documentado en diversos estudios. Por ejemplo, se ha reportado una relación entre el estrés psicosocial, abuso sexual, físico y/o verbal, condiciones de cuidado negligente durante la infancia y el desarrollo de trastornos neuropsiquiátricos como depresión, ansiedad, psicosis, trastorno por estrés postraumático y vulnerabilidad al abuso de sustancias en edades adultas (Anda et al., 2006). Estas entidades nosológicas no sólo no son excluyentes, sino también comórbidas. Así, es frecuente la comorbilidad entre los trastornos afectivos y ansiedad, y también es frecuente el trastorno por uso de sustancias (TUS) y otro u otros trastornos psiquiátricos (Craske et al., 2017; Kessler et al., 2005).

Algunos estudios, como el de Huang et al. (2011), han mostrado que, en Estados Unidos, del 31. 9\% de personas que dijeron haber sufrido algún tipo de maltrato en la infancia, el de mayor prevalencia fue el cuidado negligente, con $13.1 \%$. En este estudio analizaron el efecto de varios tipos de maltrato en la infancia sobre el uso de drogas ilícitas y problemas relacionados a su consumo. De las personas que reportaron haber vivido con cuidado negligente, el $38.9 \%$ dijeron haber usado drogas ilícitas el año anterior a la encuesta, $27.7 \%$ hicieron uso de drogas ilícitas 30 días antes de la encuesta y $15.2 \%$ tuvieron problemas relacionados con drogas el año anterior a la aplicación de la encuesta. Todos estos porcentajes fueron significativamente mayores a los reportados por personas que crecieron bajo cuidado óptimo (Huang et al., 2011). 
En la década de los 90, Widom et al. (1999) mostraron que las personas que recordaban haber tenido algún tipo de maltrato en la infancia (aun cuando no hubiera un registro oficial ante las autoridades) desarrollaron un TUS comparado con quienes no recordaban haberlo tenido (a pesar de que hubiera un registro oficial de ello) (Widom et al., 1999). Estos hallazgos sugieren que la interpretación de las vivencias como adversas es más impactante para el uso de sustancias que la vivencia real. Este factor es muy importante, ya que la teoría de la terapia cognitivo conductual (Beck y Wynnewood, 1964), que es la que más éxito tiene en rehabilitar a una persona adicta, considera que las distorsiones cognitivas (interpretación errónea de los hechos) son lo que más impacta a una persona para desarrollar diversos tipos de trastornos psiquiátricos, incluido el TUS.

Más adelante, Thornberry et al. (2010) contrastaron el impacto del maltrato en la infancia y en la adolescencia. El maltrato en la infancia mostró tener repercusiones en mediciones relacionadas con depresión y abuso de sustancias, mientras que el maltrato en la adolescencia mostró tener mayor impacto en medidas de conducta criminal, abuso de sustancias (marihuana, heroína, entre otras), conductas sexuales de riesgo y pensamientos suicidas (Thornberry et al., 2010). Si bien el maltrato en cualquier etapa de la vida se relaciona con el consumo de drogas ilícitas en la adultez, el impacto de dicha experiencia está en función de la etapa en que se sufrió maltrato. En este estudio, el maltrato en la adolescencia es el que mostró mayor efecto en el abuso de sustancias. Nuevamente, puede ser por la interpretación que el sujeto hace del evento que le ocurre y juzga la intensidad de la negatividad con diferente sensibilidad que lo que los observadores externos pueden constatar. Esta interpretación catastrofizante marca su relación con el abuso de sustancias.

Oshri et al. (2011) añadieron información al efecto del impacto del maltrato en la infancia, mostrando que el $27 \%$ de la población adolescente que reportó haber sufrido maltrato presentó al menos un síntoma de abuso y dependencia del cannabis (ADC). En contraste, en el grupo sin maltrato en la infancia (cuidado óptimo), el porcentaje fue menor, $15 \%$, presentando cuando mucho un síntoma de ADC (Oshri et al., 2011). 
Estos hallazgos sugieren que a pesar de que hay una influencia de las relaciones sociales estresantes para el desarrollo del TUS, también hay un componente genético que se expresa a pesar de tener óptimas condiciones de crianza.

\section{La soledad, el rechazo y el uso de sustancias}

El sentimiento de soledad, que la definimos operativamente como el convencimiento de no contar con una red de apoyo afectivo, ha evolucionado como un seguro de protección que al activarse aumenta la motivación para buscar a alguien con quien convivir, de tal forma que el sentimiento de soledad desaparezca (Cacciopo y Patrick, 2008). Como el hambre motiva a comer, la soledad señala un deterioro o una pérdida de las relaciones psicosociales y motiva la búsqueda de su restablecimiento o su fortalecimiento. En este contexto, la percepción del rechazo, la soledad, se vive como una experiencia altamente estresante (Campagne, 2019).

Maurage y su grupo (Maurage et al., 2012), utilizando Cyberball, estudiaron las zonas del cerebro humano que se activan cuando la persona es rechazada. Es un juego de pelota computarizado en el que el sujeto en estudio juega lanzando una pelota a otros dos jugadores que están representados digitalmente en la pantalla. El sujeto en estudio está adentro de un resonador (fMRI). El juego está preprogramado en distintos bloques, en donde el participante primero es observador, en el siguiente bloque es incluido en el juego, después es excluido y, finalmente, es reintegrado. En hombres diagnosticados con dependencia al alcohol en su tercera semana de desintoxicación, se observó una mayor activación del LOBIN y menor activación de la CPFvm cuando el sujeto fue excluido. Los sujetos relataron emociones negativas, las cuales correlacionaron positivamente con la actividad del LOBIN y negativamente con la actividad de la corteza prefrontal medial ventral (CPFvm). Posteriormente, Woo et al. (2014) mostraron cómo otras regiones cerebrales como la circunvolución frontal inferior (CFI), CPFvm, CPFdm, la circunvolución del cíngulo 
anterior pregenual (CCApg), la UTP y el precúneo (PC) se activan ante la percepción del rechazo (Woo et al., 2014).

A pesar de que ambos estudios no concuerdan en todas las regiones cerebrales involucradas, podemos sugerir que el sentimiento de soledad tiene su propia red que necesariamente comparte estructuras con otras como el cerebro social y el sistema de defensa, como hemos discutido. Otras investigaciones han mostrado que los sentimientos de soledad a lo largo del tiempo causan el mismo daño que tener presión arterial alta, falta de ejercicio, obesidad o fumar (Holt-Lunstad et al., 2015; House et al., 1988). Estudios recientes han mostrado que el aislamiento inducido por la pandemia de COVID-19 aumenta el consumo de alcohol, opioides y marihuana (Killgore et al., 2021; Mallet et al., 2021).

El consumo compulsivo de sustancias afecta la habilidad de funcionar en un contexto social (Ersöğütçü y Karakaş, 2016), lo cual puede llevar a la persona a la marginalización, al aislamiento y a la soledad, promoviendo el mantenimiento del TUS y trastornos psiquiátricos comórbidos (Laudet, 2008). Asimismo, la actitud del grupo que devalúa a las personas que padecen TUS causa sentimientos de rechazo, los cuales promueven la recaída (Glass et al., 2013). Lamentablemente, la estigmatización de personas con TUS la exhiben aún profesionales de la salud mental, lo cual interfiere con el tratamiento efectivo de dichos pacientes (Van Boekel et al., 2013). Observamos también que aquellas personas etiquetadas como adictos, alcohólicos, marihuanos, entre otras etiquetas, son fácilmente asociados a actividades criminales, inmorales o enfermizas, fomentando de esta manera la marginación y manteniendo o agravando el TUS. Es posible, como una especulación, decir que la falta del reforzamiento social, como serían la aceptación, la inclusión y la dignificación de la persona, propicia el abuso de sustancias para facilitar al sistema de reforzamiento y, simultáneamente, reducir la actividad del sistema de defensa en una forma que podría incluso considerarse una automedicación. 
El sociólogo Erwin Goffman fue unos de los pioneros en postular que la estigmatización de personas con un trastorno mental es un dilema que debemos abordar como grupo. Goffman definió al estigma como la relación que la gente común encuentra entre un atributo del individuo y un estereotipo conformado de características no deseadas (Goffman, 1963). Esta concepción relacional del estigma sugiere que la imposición de etiquetas determina cómo los otros perciben y tratan al estigmatizado. Autores posteriores a Goffman se enfocaron en la consecuencia de la estigmatización como un factor que contribuye al desarrollo y mantenimiento de trastornos mentales (Link et al., 1989; Scheff, 1999). La percepción de ser estigmatizado por padecer un TUS está correlacionada con una mayor comorbilidad psiquiátrica, lo cual podría ser consecuencia de un menor apoyo social (Glass et al., 2013). Además, los individuos etiquetados desarrollan estrategias de afrontamiento o evitación de interacciones porque anticipan ser discriminados y devaluados, lo que potencialmente los hace sentir incómodos o amenazados (Link et al., 2009). Congruente con estos hallazgos, la literatura científica indica que la internalización del estigma relacionado al trastorno mental tiene una robusta correlación negativa con emociones positivas, v.gr, esperanza, autoestima, empoderamiento y apoyo social, mientras que se correlaciona de manera positiva con la severidad de los síntomas (Livingston y Boyd, 2010).

Se ha propuesto que las relaciones psicosociales positivas impactan en el bienestar físico y mental del humano al promover conductas adaptativas, además de que las interacciones psicosociales exitosas ayudan a evitar una adicción a sustancias debido a que son un reforzador positivo que compite contra el reforzamiento inducido por el abuso de sustancias (Marín-Navarrete et al., 2013; Niño et al., 2016). Consecuentemente, si dichas relaciones psicosociales son negativas, por ejemplo, si el sujeto se percibe agredido o excluido de su grupo y sin red de apoyo, buscará otros reforzadores, como es el abuso de sustancias (Osgood et al., 2014). En este contexto, se ha reportado que los adolescentes con menor interacción con sus pares tienen mayor probabilidad de consumir tabaco (Osgood et al., 2014; Oshri et al., 
2011). Niño et al. (2016) han mostrado que, si un adolescente se percibe solo, la probabilidad de consumir alcohol y tabaco aumenta. Este efecto es específico de aquellos que muestran poca o nula interacción con otras personas, aunque esta actitud frecuentemente no es voluntaria (Niño et al., 2016). Además, se ha documentado que vivir en situaciones sociales que el sujeto considera estresantes debilita la fortaleza de la abstinencia a sustancias, por lo que aumenta la probabilidad de una recaída (Osgood et al., 2014). Todas estas investigaciones hacen notar que las relaciones sociales negativas tienen un impacto facilitador sobre las conductas relacionadas al consumo de drogas.

\section{La inclusión y la dignificación del adicto a sustancias}

Sin embargo, las relaciones sociales positivas pueden apoyar a una persona a mantenerse en abstinencia. Por ejemplo, Thomas et al. (2019) mostraron que la existencia de una red de apoyo que incluya a la familia y a los amigos, y la actitud positiva del proveedor de salud, médico, terapeuta y otros, es muy importante para el inicio y mantenimiento de la abstinencia. De hecho, la teoría del capital de recuperación (recovery capital; Irvine et al., 2001) sugiere que conforme más capital social (la suma de sus relaciones sociales, v. gr., familia y amigos), capital físico (la suma de sus posesiones, v. gr., dinero y propiedad), capital humano (habilidades y condición física, v. gr., aspiraciones y recursos cognitivos) y capital cultural (valores y creencias) tenga la persona, sus perspectivas de recuperación son mejores (Cloud y Granfield, 2008). Dentro del capital social, podemos incluir las congregaciones religiosas que comparten una forma especial de convivencia, con normas y principios religiosos, la liturgia, que hacen que el humano tenga autocontrol sobre diversos aspectos de su vida.

\section{El cerebro místico/religioso y el uso de sustancias}

Debido a que el misticismo o espiritualidad es un concepto personal, una interpretación idiosincrática de hechos o eventos, no creemos que podamos manejarla de una forma general para entender cómo facilita la abstinencia en personas adictas. Sin embargo, la religiosidad es más tangible. La religiosidad es la práctica 
de las normas de la liturgia y ésta sí nos permite ver cómo influencia el desarrollo y permanencia del TUS. Una pregunta legítima sería por qué la religiosidad tendría que ver con el TUS. Como un aspecto contextual, consideremos que el Pew Research Center en una encuesta foro sobre religión y vida pública (PewReligion, 2014) ha estimado que el $84 \%$ de la población universal es religiosa. Esto sugiere que una parte de las personas que consumen sustancias (con o sin TUS) son religiosos.

La religiosidad se expresa siguiendo las normas que dicta la liturgia de la congregación, con el convencimiento de que dichas normas han sido dictadas por su dios. Independientemente de creer en la existencia de una divinidad, parece que el cerebro tiene sistemas encargados de producir una creencia en lo sagrado, el cerebro místico (Cristofori et al., 2016). Esto es solamente obvio, ya que no hay pensamiento o emoción que no sea generada por el cerebro. Además, algunos investigadores han sugerido la existencia de genes que regulan esta creencia (Hamer y Copeland, 1999; Wang et al., 2019), por lo que tendríamos que aceptar que creer en lo divino, o ser místico, o religioso está genéticamente facilitado. La pertinencia de la creencia en lo sagrado en la cognición y en la adaptación del humano al ambiente es que promueve (1) la motivación de continuidad; es decir, la persecución del objetivo y (2) la motivación intrínseca al sujeto, el esfuerzo, en situaciones de extrema adversidad. Por ejemplo, cuando el sujeto creyente o religioso enfrenta una amenaza, es decir, un estímulo que el sujeto considera que supera todos sus recursos, solicita la intervención divina con la expectativa de enfrentar con mayor probabilidad de éxito a dicho estímulo. La persona que valora ciertos hechos y estímulos como sagrados considera que la divinidad tiene sus demandas, por lo que es necesario portarse bien para estar en la gracia de la divinidad y, por lo mismo, recibir su protección en todo momento. ¿Qué es portarse bien? Está descrito en la liturgia de cualquiera de las religiones y lo que comparten dichas liturgias es, esencialmente, la demanda del autocontrol del sujeto. No matarás, no fornicarás, no robarás, honrarás a tu padre y a tu madre, entre otras, son condiciones que la divinidad impone para otorgar su protección y su premiación máxima que es el acceso al paraíso. El no, en 
estas aseveraciones, implica autocontrol. Es volitivo y, por lo mismo, depende de que el sujeto tenga claras las consecuencias de no obedecer el mandato.

El cerebro de las personas que transitan por un estado místico es particularmente diferente a cuando están en otros estados. Un estado místico es considerado como el momento en que las personas sienten que tienen una comunión con la divinidad en el plano terrenal. A pesar de la tremenda subjetividad del evento, se ha mostrado que el PC derecho, el lóbulo temporal derecho, el LOBIN y la CPFdm están involucrados en producir este estado (Szerman, 2016). Este cerebro místico ha generado normas y ritos que permiten la glorificación de lo divino que incluyen el autocontrol de conductas que el grupo considera dañinas para el sujeto y para el propio grupo. Por lo tanto, encontramos que las personas creyentes de lo divino utilizan la liturgia de su religión para autocontrolarse. Es decir, muchas personas no consumen sustancias porque la religión lo prohíbe y muchas otras logran autocontrolar su dependencia gracias a que se encomiendan a una divinidad, v.gr., los doce pasos de AA.

En este contexto, discutimos el trabajo de Schoenthaler et al. (2015) quienes evaluaron a 2947 personas que habían sido sometidas a rehabilitación de un TUS. A pesar de que ellos refieren que evaluaron espiritualidad, en realidad evaluaron religiosidad. Por ejemplo, evaluaron la fortaleza de sus creencias religiosas, la frecuencia con la que asistían a la iglesia, si leían libros religiosos, si veían programas religiosos y si meditaban/oraban. Todos estos parámetros, de acuerdo con un análisis de regresión logística, contribuyeron a que las personas retrasaran la recaída o no la tuvieran para nada en el consumo de cocaína, heroína, alcohol y marihuana (Schoenthaler et al., 2015). Asimismo, Jalali et al. (2019), evaluando una muestra de 117 usuarios de metanfetamina, han reportado que la baja percepción de apoyo social y baja religiosidad son factores de riesgo para el consumo de metanfetamina. No solamente eso, sino que observaron que incluir a las personas que están siendo tratadas con metadona, por dependencia a opioides, a un grupo de apoyo religioso (30 personas fueron canalizadas al grupo religioso) fortalecieron sus deseos de 
mantener la abstinencia, comparados con los que no fueron apoyados por el grupo religioso (otras 30 personas; Jalali et al., 2019). En el más reciente estudio consultado (Oliveira et al., 2020), el cuidado espiritual de pacientes internados en una clínica de rehabilitación facilitó su recuperación desafortunadamente no hay un seguimiento en ninguno de estos estudios.

Es posible que una persona que se acerca a una comunidad religiosa para fortalecer su deseo de rehabilitarse de un TUS solamente está integrándose a una comunidad con la que establece relaciones psicosociales y, por la naturaleza de ésta, reciba aceptación e integración al grupo y se le involucre en actividades prosociales. Estos aspectos de interacción social proveen a la persona de aceptación, inclusión y dignificación.

\section{Discusión}

A pesar de que la dependencia a sustancias es un problema multifactorial, todas estas circunstancias convergen en una conducta que a pesar de que es extremadamente obvia es imprescindible de mencionar para entender cómo se genera la adicción: la persona tiene que consumir la droga frecuentemente y en altas cantidades. En esta revisión, esperamos haber mostrado que para que una persona consuma una droga, o varias, hasta llegar a la adicción, debe tener una vulnerabilidad genética o tener los cambios epigenéticos cerebrales inducidos por interacciones psicosociales negativas, que finalmente son las que propician y mantienen la dependencia. Es decir, traer una carga genética que vulnere a la persona no es suficiente para que se instale la dependencia, es necesario que las interacciones psicosociales sean adversas, desde antes de que se vuelva adicto. Particularmente, la infancia y la adolescencia son periodos críticos en que el abuso de cualquier tipo, o la negligencia, fomentan las condiciones para el posterior consumo de sustancias.

La actitud de las personas no adictas frente a los adictos es de baja tolerancia, estigmatización y aislamiento social. Esto puede llevar a la persona a un sentimiento de soledad, y a propiciar la búsqueda de grupos marginados para incluirse en ellos, lo que facilita el consumo de sustancias. Es decir, la falta del 
apoyo social, de la red de apoyo, por ejemplo, de la familia, o de congregaciones que comparten una creencia religiosa y una liturgia, facilita la permanencia de la persona adicta en el consumo. En este contexto, se vuelve crucial psicoeducar a la población no adicta para sensibilizarlos y que acepten que la adicción a sustancias es una enfermedad del cerebro, facilitada y mantenida por el rechazo y la estigmatización del usuario.

En este contexto, es importante reconocer que la inclusión de esta persona adicta a un grupo de apoyo, en el que se privilegie el fortalecimiento de sus recursos cognitivos, físicos y espirituales, resulta crucial para el éxito de una terapia farmacológica/psicoterapéutica y, por lo mismo, para su rehabilitación. La interacción con personas que respetan y fomentan la espiritualidad permite el desarrollo de un convencimiento de pertenencia que restablece la dignidad del paciente. Es decir, el restablecimiento de su valía.

Nosotros, los autores, reconocemos que la cantidad de estudios que apoyan nuestra revisión es baja. Así que esta revisión si algo tiene son limitaciones en el número de estudios que la apoyen, pero es una avenida que tenemos que explorar con más investigación, particularmente porque hoy por hoy, el éxito que tenemos en rehabilitar a los pacientes que lo solicitan es bajo.

\section{Conclusiones}

En conclusión, podemos decir que, además del tratamiento farmacológico y psicoterapéutico, las posibilidades de que un paciente se rehabilite aumentan cuando se incluye en su cuidado su integración a grupos de apoyo y se fomenta su espiritualidad.

\section{Conflicto de intereses}

Los autores no tienen conflicto de intereses que declarar. 


\section{Financiamiento}

Este trabajo se realizó con apoyo de la Dirección General de Asuntos del Personal Académico-Programa de Apoyo a Proyectos de Investigación e Innovación Tecnológica, Universidad Nacional Autónoma de México (DGAPA-PAPIIT-UNAM), Nm.: IN218620, IA205218, IN217221 a OPG, MMD, y AERC.

\section{Referencias}

Anda, R. F., Felitti, V. J., Bremmer, J. D., Walker, J. D., Whitfield, C., Perry, B. D., Dube, S. R. y Giles, W. (2006). The Enduring Effects of Abuse and Related Adverse Experiences in Childhood: A Convergence of Evidence from Neurobiology and Epidemiology. European Archives of Psychiatry and Clinical Neuroscience, 256(3), 174-186. https://doi.org/10.1007/s00406-005-0624-4

Asok, A., Leroy, F., Rayman, J. B. y Kandel, E. R. (2019). Molecular Mechanisms of the Memory Trace. Trends in Neurosciences, 42(1), 14-22. https://doi.org/10.1016/j.tins.2018.10.005

Bandura, A. y Walters, R. H. (1974). Aprendizaje social y desarrollo de la personalidad (Vol. 1974). Alianza editorial.

Beck, A. T, Rush, A. J., Shaw, B. F. y Emery, G. (1979). Cognitive Therapy of Depression. New York: Guilford Press.

Beck, A. y Wynnewood, P. (1964). Thinking and Depression. Archives of General Psychiatry, 10, 561571.

Berridge, K. C. (2018). Evolving Concepts of Emotion and Motivation. In Frontiers in Psychology (Vol. 9, Issue SEP, pp. 1-20). https://doi.org/10.3389/fpsyg.2018.01647

Bifulco, A., Brown, G. W. y Adler, Z. (1991). Early Sexual Abuse and Clinical Depression in Adult Life. British Journal of Psychiatry, 159(JUL), 115-122.

Blakemore, S. J. (2010). The Developing Social Brain: Implications for Education. Neuron, 65(6), 744747. https://doi.org/10.1016/j.neuron.2010.03.004 
Blakemore, S. J., Den Ouden, H., Choudhury, S. y Frith, C. (2007). Adolescent Development of the Neural Circuitry for Thinking about Intentions. Social Cognitive and Affective Neuroscience, 2(2), 130-139. https://doi.org/10.1093/scan/nsm009

Blakemore, S. J. y Frith, U. (2004). How does the brain deal with the social world? NeuroReport, 15(1), 119-128. https://doi.org/10.1097/00001756-200401190-00024

Cacciopo, J. y Patrick, W. (2008). Loneliness: Human Nature and the need for Social Connection (1st ed.). W W Norton y Co.

Campagne, D. M. (2019). Stress and Perceived Social Isolation (loneliness). Archives of Gerontology and Geriatrics, 82(June 2018), 192-199. https://doi.org/10.1016/j.archger.2019.02.007

Cannon, W. B. (1914). The Interrelations of Emotions as Suggested by Recent Physiological Researches. The American Journal of Psychology, 25(2), 256. https://doi.org/10.2307/1413414

Cloud, W. y Granfield, R. (2008). Conceptualizing Recovery Capital: Expansion of a Theoretical Construct. Substance Use and Misuse, 43(12-13), 1971-1986. https://doi.org/10.1080/10826080802289762

Conway, K. P., Compton, W., Stinson, F. S. y Grant, B. F. (2006). Lifetime Comorbidity of DSM-IV Mood and Anxiety Disorders and Specific Drug Use Disorders. The Journal of Clinical Psychiatry, 67(02), 247-258. https://doi.org/10.4088/jcp.v67n0211

Craske, M. G., Stein, M. B., Eley, T. C., Milad, M. R., Holmes, A., Rapee, R. M. y Wittchen, H.-U. (2017). Anxiety Disorders. Nature Reviews Disease Primers, 3(1), 17024.

\section{https://doi.org/10.1038/nrdp.2017.24}

Cristofori, I., Bulbulia, J., Shaver, J. H., Wilson, M., Krueger, F. y Grafman, J. (2016). Neural Correlates of Mystical Experience. Neuropsychologia, 80, 212-220. https://doi.org/10.1016/j.neuropsychologia.2015.11.021 
Critchley, H. y Garfinkel, S. (2014). Neural Correlates of Fear: Insights from Neuroimaging. Neuroscience and Neuroeconomics, 111. https://doi.org/10.2147/nan.s35915

Den Ouden, H. E. M., Frith, U., Frith, C. y Blakemore, S. J. (2005). Thinking about Intentions. NeuroImage, 28(4), 787-796. https://doi.org/10.1016/j.neuroimage.2005.05.001

Dunbar, R. I. M. (2009). The Social Brain Hypothesis and its Implications for Social Evolution. Annals of Human Biology, 36(5), 562-572. https://doi.org/10.1080/03014460902960289

Ekman, P. (1993). Facial Expression and Emotion. In American Pshychologist Association (pp. 384-392).

Ersöğütçü, F. y Karakaş, S. A. (2016). Social Functioning and Self-Esteem of Substance Abuse Patients. Archives of Psychiatric Nursing, 30(5), 587-592. https://doi.org/10.1016/j.apnu.2016.03.007

Farroni, T., Johnson, M. H., Menon, E., Zulian, L., Faraguna, D. y Csibra, G. (2005). Newborns’ Preference for Face-relevant Stimuli: Effects of contrast polarity. Proceedings of the National Academy of Sciences of the United States of America, 102(47), 17245-17250. https://doi.org/10.1073/pnas.0502205102

Frith, C. D. y Frith, U. (2007). Social Cognition in Humans. Current Biology, 17(16), 724-732. https://doi.org/10.1016/j.cub.2007.05.068

Glass, J. E., Mowbray, O. P., Link, B. G., Kristjansson, S. D. y Bucholz, K. K. (2013). Alcohol Stigma and Persistence of Alcohol and Other Psychiatric Disorders: A Modified Labeling Theory approach. Drug and Alcohol Dependence, 133(2), 685-692. https://doi.org/10.1016/j.drugalcdep.2013.08.016

Goffman, E. (1963). Stigma: Notes on the Management of Spoiled Identity. In Penguin Books (Issue 1). https://doi.org/10.2307/2575995

Gogtay, N., Giedd, J. N., Lusk, L., Hayashi, K. M., Greenstein, D., Vaituzis, A. C., Nugent, T. F., Herman, D. H., Clasen, L. S., Toga, A. W., Rapoport, J. L. y Thompson, P. M. (2004). Dynamic Mapping of Human Cortical Development During Childhood Through Early Adulthood. Proceedings of the 
National Academy of Sciences of the United States of America, 101(21), 8174-8179. https://doi.org/10.1073/pnas.0402680101

Grupe, D. W. y Nitschke, J. B. (2013). Uncertainty and Anticipation in Anxiety: An Integrated Neurobiological and Psychological Perspective. Nature Reviews Neuroscience, 14(7), 488-501. https://doi.org/10.1038/nrn3524

Hamer, D. y Copeland, P. (1999). Living with Our Genes: With Their Matter More Than You Think. Ancor.

Hebb, D. O. (1949). The Organization of Behavior; A Neuropsychological Theory. J. Wiley; Chapman y Hall.

Heim, C., Shugart, M., Craighead, W. E. y Nemeroff, C. B. (2010). Neurobiological and Psychiatric Consequences of Child Abuse and Neglect. Developmental Psychobiology, 52(7), 671-690. https://doi.org/10.1002/dev.20494

Holt-Lunstad, J., Smith, T. B., Baker, M., Harris, T. y Stephenson, D. (2015). Loneliness and Social Isolation as Risk Factors for Mortality: A Meta-Analytic Review. Perspectives on Psychological Science, 10(2), 227-237. https://doi.org/10.1177/1745691614568352

House, J. S., Landis, K. R., Umberson, D., House, J. S., Landis, I. A. R. L. R. y Umberson, D. (1988). Social Relationships and Health. Science, 241(4865), 540-545.

\section{https://doi.org/10.1126/science.3399889}

Huang, S., Trapido, E., Fleming, L., Arheart, K., Crandall, L., French, M., Malcolm, S. y Prado, G. (2011). The Long-term Effects of Childhood Maltreatment Experiences on Subsequent Illicit Drug Use and Drug-related Problems in Young Adulthood. Addictive Behaviors, 36(1-2), 95-102. https://doi.org/10.1016/j.addbeh.2010.09.001

Irvine, L., Granfield, R. y Cloud, W. (2001). Coming Clean: Overcoming Addiction without Treatment. 
Contemporary Sociology, 30(1), 86. https://doi.org/10.2307/2654369

Jalali, A., Behrouzi, M. K., Salari, N., Bazrafshan, M.-R. y Rahmati, M. (2019). The Effectiveness of Group Spiritual Intervention on Self-esteem and Happiness Among Men Undergoing Methadone Maintenance Treatment. Current Drug Research Reviews, 11(1), 67-72. https://doi.org/10.2174/1874473711666180510164420

Kandel, E., y Schwartz, J. (1982). Molecular iology of Learning: Mmodulation of ransmitter Release. Science, 218(4571), 433-443. https://doi.org/10.1126/science.6289442

Kessler, R. C., Chiu, W. T., Demler, O. y Walters, E. E. (2005). Prevalence, Severity, and Comorbidity of 12-Month DSM-IV Disorders in the National Comorbidity Survey Replication. Archives of General Psychiatry, 62(6), 617. https://doi.org/10.1001/archpsyc.62.6.617

Killgore, W. D. S., Cloonan, S. A., Taylor, E. C., Lucas, D. A. y Dailey, N. S. (2021). Alcohol Dependence During COVID-19 Lockdowns. Psychiatry Research, 296. https://doi.org/10.1016/j.psychres.2020.113676

King, M. F. y Bruner, G. C. (2000). Social Desirability Bias: A Neglected Aspect of Validity Testing. Psychology and Marketing, 17(2), 79-103. https://doi.org/10.1002/(SICI)1520-

\section{$\underline{6793(200002) 17: 2<79:: A I D-M A R 2>3.0 . C O ; 2-0}$}

Kohls, G., Perino, M. T., Taylor, J. M., Madva, E. N., Cayless, S. J., Troiani, V., Price, E., Faja, S., Herrington, J. D. y Schultz, R. T. (2013). The Nucleus Accumbens Is Involved in Both the Pursuit of Social Reward and the Avoidance of Social Punishment. Neuropsychologia, 51(11), 2062-2069. https://doi.org/10.1016/j.neuropsychologia.2013.07.020

Kumaran, D., Melo, H. L. y Duzel, E. (2012). The Emergence and Representation of Knowledge about Social and Nonsocial Hierarchies. Neuron, 76(3), 653-666.

https://doi.org/10.1016/j.neuron.2012.09.035 
Laudet, A. B. (2008). The road to recovery: Where Are Ee Going and How do We Get There? Empirically Driven Conclusions and Future Directions for Service Development and Research. In Substance Use and Misuse (Vol. 43, Issues 12-13, pp. 2001-2020). Taylor and Francis Inc.

https://doi.org/10.1080/10826080802293459

Lewis, P. A., Birch, A., Hall, A. y Dunbar, R. I. M. (2017). Higher Order Intentionality Tasks are Cognitively more Demanding. Social Cognitive and Affective Neuroscience, 12(7), 1063-1071. https://doi.org/10.1093/scan/nsx034

Link, B. G., Mirotznik, J. y Cullen, F. T. (2009). The Effectiveness of Stigma Coping Orientations : Can Negative Consequences of Mental Illness Labeling be Avoided? Health (San Francisco), 32(3), 302320.

Link, B. G., Cullen, F. T., Struening, E., Shrout, P. E., Dohrenwend, P., Link, B. G., Cullen, F. T., Struening, E. y Shrout, P. E. (1989). A Modified Labeling Theory Approach to Mental Disorders : An Empirical Assessment. American Sociological Review, 54(3), 400-423.

Livingston, J. D. y Boyd, J. E. (2010). Correlates and Consequences of Internalized Stigma for People Living with Mental Illness: A Systematic Review and Meta-analysis. Social Science and Medicine, 71(12), 2150-2161. https://doi.org/10.1016/j.socscimed.2010.09.030

Mallet, J., Dubertret, C. y Le Strat, Y. (2021). Addictions in the COVID-19 Era: Current Evidence, Future Perspectives a Comprehensive review. Progress in Neuro-Psychopharmacology and Biological Psychiatry, 106(January), 110070. https://doi.org/10.1016/j.pnpbp.2020.110070

Marín-Navarrete, R., Benjet, C., Borges, G., Eliosa-Hernández, A., Nanni-Alvarado, R., Ayala-Ledesma, M., Fernández-Mondragón, J. y Medina-Mora, M. E. (2013). Comorbilidad de los trastornos por consumo de sustancias con otros trastornos psiquiátricos en Centros Residenciales de Ayuda-Mutua para la Atención de las Adicciones. Salud Mental, 36(6), 471-479. https://doi.org/10.17711/SM.0185- 


\section{$\underline{3325.2013 .057}$}

Maurage, P., Joassin, F., Philippot, P., Heeren, A., Vermeulen, N., Mahau, P., Delperdange, C., Corneille, O., Luminet, O. y De Timary, P. (2012). Disrupted Regulation of Social Exclusion in AlcoholDependence: An fMRI study. Neuropsychopharmacology, 37(9), 2067-2075.

\section{https://doi.org/10.1038/npp.2012.54}

McEwen, B. S. (2016). In Pursuit of Resilience: Stress, Epigenetics, and Brain Plasticity. Annals of the New York Academy of Sciences, 1373(1), 56-64. https://doi.org/10.1111/nyas.13020

Niño, M. D., Cai, T. y Ignatow, G. (2016). Social Isolation, Drunkenness, and Cigarette Use among Adolescents. Addictive Behaviors, 53, 94-100. https://doi.org/10.1016/j.addbeh.2015.10.005

Oliveira, C. P. de, Calixto, A. M., Disconzi, M. V., Pinho, L. B. de y Camatta, M. W. (2020). Spiritual Care Performed in a Drug User Clinic. Revista Gaucha de Enfermagem, 41(spe), e20190121. https://doi.org/10.1590/1983-1447.2020.20190121

Osgood, D. W., Feinberg, M. E., Wallace, L. N. y Moody, J. (2014). Friendship Group Position and Substance Use. Addictive Behaviors, 39(5), 923-933. https://doi.org/10.1016/j.addbeh.2013.12.009

Oshri, A., Rogosch, F. A., Burnette, M. L. y Cicchetti, D. (2011). Developmental Pathways to Adolescent Cannabis Abuse and Dependence: Child Maltreatment, Emerging Personality, and Internalizing versus Externalizing Psychopathology. Psychology of Addictive Behaviors, 25(4), 634-644. https://doi.org/10.1037/a0023151

Petanjek, Z., Judaš, M., Šimić, G., Rašin, M. R., Uylings, H. B. M., Rakic, P. y Kostović, I. (2011). Extraordinary Neoteny of Synaptic Spines in the Human Prefrontal Cortex. Proceedings of the National Academy of Sciences of the United States of America, 108(32), 13281-13286. https://doi.org/10.1073/pnas.1105108108

PewReligion. (2014). Una nueva encuesta de gran escala explora el cambio en la identidad religiosa de 
los latinos en los Estados Unidos. Pew Reserch Center, Religion y Public Life.

https://www.pewforum.org/2014/05/09/una-nueva-encuesta-de-gran-escala-explora-el-cambio-en-la$\underline{\text { identidad-religiosa-de-los-latinos-en-los-estados-unidos/ }}$

Prospero-Garcia, O. E., Ruiz-Contreras, A. E., Morelos, J., Herrera-Solis, A. y Mendez-Díaz, M. (2021).

Fragility of Reward vs Antifragility of Defense Brain Systems in Drug Dependence. Social

Neuroscience, 1-8. https://doi.org/10.1080/17470919.2021.1876759

Regier, D. A., Farmer, M. E., Rae, D. S., Locke, B. Z., Keith, S. J., Judd, L. L. y Goodwin, F. K. (1990).

Comorbidity of Mental Disorders With Alcohol and Other Drug Abuse: Results From the

Epidemiologic Catchment Area (ECA) Study. Journal of the American Medical Association, 264(19), 2511-2518. https://doi.org/10.1001/jama.1990.03450190043026

Rizzolatti, G. y Sinigaglia, C. (2016). The Mirror Mechanism: A Basic Principle of Brain Function. Nature Reviews Neuroscience, 17(12), 757-765. https://doi.org/10.1038/nrn.2016.135

Scheff, T. J. (1999). Being Mentally Ill: A Sociological Theory. In Journal of Health and Social Behavior (Issue 3). Aldine Transaction.

Schoenthaler, S. J., Blum, K., Braverman, E. R., Giordano, J., Thompson, B., Oscar-Berman, M., Badgaiyan, R. D., Madigan, M. A., Dushaj, K., Li, M., Demotrovics, Z., Waite, R. L. y Gold, M. S. (2015). NIDA-Drug Addiction Treatment Outcome Study (DATOS) Relapse as a Function of Spirituality/Religiosity. Journal of Reward Deficiency Syndrome, 01(01), 36-45. doi:

10.17756/jrds.2015-007

Solomon, R. L. y Corbit, J. D. (1974). An Opponent-process Theory of Motivation. I. Temporal dynamics of affect. Psychological Review, 81(2), 119-145.

Stiles, J. (2017). Principles of Brain Development. Wiley Interdisciplinary Reviews: Cognitive Science, 8(1-2), e1402. https://doi.org/10.1002/wcs.1402 
Stodel, M. (2015). But what will People Think? Getting Beyond Social Desirability Bias by Increasing Cognitive Load. International Journal of Market Research, 57(2), 313-321. https://doi.org/10.2501/IJMR-2015-024

Szerman, N. (2016). Introducción a la conceptualización de la patología dual. Protocolos de Intervención de Patología Dual, 15. https://patologiadual.es/wp-content/uploads/2019/02/0-intro-pdual-drszerman.pdf

Taleb, N. N. y Douady, R. (2012). Mathematical Definition, Mapping, and Detection of (Anti)Fragility. SSRN Electronic Journal, 13(11), 1677-1689. https://hal.archives-ouvertes.fr/hal01151340/document

Thomas, D. P., Davey, M. E., van der Sterren, A. E., Lyons, L., Hunt, J. M. y Bennet, P. T. (2019). Social Networks and Quitting in a National Cohort of Australian Aboriginal and Torres Strait Islander Smokers. Drug and Alcohol Review, 38(1), 82-91. https://doi.org/10.1111/dar.12891

Thornberry, T. P., Henry, K. L., Ireland, T. O. y Smith, C. A. (2010). The Causal Impact of ChildhoodLimited Maltreatment and Adolescent Maltreatment on Early Adult Adjustment. Journal of Adolescent Health, 46(4), 359-365. https://doi.org/10.1016/j.jadohealth.2009.09.011

UNICEF. (2019). Annual Report 2018: For Every Child, Every Right. https://www.unicef.org/publications UNODC. (2018). World Drug Report.

\section{https://www.unodc.org/wdr2018/prelaunch/WDR18_Booklet_4_YOUTH.pdf}

Van Boekel, L. C., Brouwers, E. P. M., Van Weeghel, J. y Garretsen, H. F. L. (2013). Stigma among Health Professionals towards Patients with Substance Use Disorders and its Consequences for Healthcare Delivery: Systematic review. Drug and Alcohol Dependence, 131(1-3), 23-35. https://doi.org/10.1016/j.drugalcdep.2013.02.018

Vicario, C. M., Rafal, R. D., Martino, D. y Avenanti, A. (2017). Core, Social and Moral Disgust are 
Bounded: A Review on Behavioral and Neural Bases of Repugnance in Clinical Disorders.

Neuroscience and Biobehavioral Reviews, 80(December 2016), 185-200.

https://doi.org/10.1016/j.neubiorev.2017.05.008

Vygotsky, L. S. (1995). Pensamiento y lenguaje. Teoría del desarrollo cultural de las funciones psíquicas. Fausto.

Wang, L., Koenig, H. G., He, Z., Sun, X., Shohaib, S. Al y Wang, Z. (2019). Religiosity and Telomere Length: Moderating Effect of Religiosity on the Relationship Between High-Risk Polymorphisms of the Apolipoprotein E and TOMM40 Gene and Telomere Length. Journal of Applied Gerontology. https://doi.org/10.1177/0733464819865415

Widom, C. S., Weiler, B. L. y Cottler, L. B. (1999). Childhood Victimization and Drug Abuse: A Comparison of Prospective and Retrospective Findings. Journal of Consulting and Clinical Psychology, 67(6), 867-880. https://doi.org/10.1037/0022-006X.67.6.867

Woo, C. W., Koban, L., Kross, E., Lindquist, M. A., Banich, M. T., Ruzic, L., Andrews-Hanna, J. R. y Wager, T. D. (2014). Separate Neural Representations for Physical Pain and Social Rejection. Nature Communications, 5(May), 1-12. https://doi.org/10.1038/ncomms6380

\footnotetext{
Cómo citar este artículo: Franco, G., Migliaro, M., Molina, L., Méndez-Díaz, M., Ruiz-Contreras, A. E., Cortés-Morelos, J., Herrera-Solís, A., \& Prospéro-García, O. E. (2021). El cerebro social y místico en el paciente dependiente de sustancias. Psicumex, 11(1), 1-31, e393. https://doi.org/10.36793/psicumex.v11i1.393
} 